\title{
DIHEDRAL ALGEBRAS ARE CYCLIC
}

\author{
PASCAL MAMMONE AND JEAN-PIERRE TIGNOL
}

\begin{abstract}
This note gives a simple proof of the following theorem of Rowen and Saltman: Every central simple algebra split by a Galois extension of rank $2 n$ ( $n$ odd) with dihedral Galois group is cyclic if the center contains a primitive nth root of unity.
\end{abstract}

The aim of this note is to provide a short conceptual proof of the following theorem of Rowen and Saltman [3].

THEOREM. Let $n$ be an odd (positive) integer and let $F$ be a field containing a primitive nth root of unity. Every central simple F-algebra split by a Galois extension of $F$ of rank $2 n$ with dihedral Galois group is also split by a cyclic extension of $F$.

The proof given here only uses basic properties of symbols and of the corestriction map, and can be adapted to the case where char $F=n$ (instead of $F$ containing a primitive $n$th root of unity), to yield a particular case of a general theorem of Albert [1].

Henceforth, we fix an odd integer $n$ and a field $F$ containing a primitive $n$th root of unity, and a Galois extension $K / F$ with dihedral Galois group generated by two elements $\sigma, \tau$ subject to the relations

$$
\sigma^{n}=1, \quad \tau^{2}=1, \quad \sigma \tau \sigma=\tau .
$$

Let $L$ be the fixed field of $\sigma$ in $K$.

LEMmA. There is an element $a \in L^{x}$ such that $K=L\left(a^{1 / n}\right)$ and $N_{L / F}(a) \in F^{x n}$.

Proof. Since $K / L$ is cyclic of rank $n$ and $L$ contains a primitive $n$th root of unity $\zeta$, one can find $\alpha \in K$ such that $K=L(\alpha)$ and $\sigma(\alpha)=\zeta \alpha$. Applying $\sigma \tau$ to both sides of this equation yields $\tau(\alpha)=\zeta \sigma \tau(\alpha)$, and it follows that $\alpha \tau(\alpha)$ is fixed under $\sigma$. This element is clearly fixed under $\tau$ too, so $\alpha \tau(\alpha) \in F^{x}$. Denoting $\alpha^{n}=a$, we have $K=L\left(a^{1 / n}\right)$ and $N_{L / F}(a)=(\alpha \tau(\alpha))^{n} \in F^{x n}$, as required. Q.E.D.

PRoOf OF THE THEOREM. Let $A$ be a central simple $F$-algebra split by $K$. By [2, Theorem 14, p. 68], $A$ decomposes as $A_{1} \otimes_{F} A_{2}$ where the degree of $A_{1}$ is a power of 2 and the degree of $A_{2}$ is odd. Both $A_{1}$ and $A_{2}$ are split by $K$, hence $A_{1}$ is split by $L$, and it suffices to prove that $A_{2}$ is split by a cyclic extension of $F$.

Received by the editors August 8, 1986.

1980 Mathematics Subject Classification (1985 Revision). Primary 16A39, 12 E15.

Key' words and phrases. Central simple algebra, cyclic algebra, corestriction.

Supported in part by the F.N.R.S. 
Since the degree of $A_{2}$ is odd, $A_{2}$ is similar (in the Brauer group of $F$ ) to an even power of itself: let $A_{2} \sim A_{2}^{2 m}$ for some integer $m$. By [2, Lemma 9, p. 54], $A_{2}^{2} \sim \operatorname{Cor}_{L / F}\left(A_{2} \otimes_{F} L\right)$, hence raising both sides to the $m$ th power, we get

$$
A_{2} \sim \operatorname{Cor}_{L / F}\left(A_{2}^{m} \otimes_{F} L\right) \text {. }
$$

Now, since $K=L\left(a^{1 / n}\right)$ splits $A_{2}$, hence also $A_{2}^{m} \otimes L$, there exists $b \in L^{x}$ such that $A_{2}^{m} \otimes L$ is similar to the symbol algebra $(a, b)$ of degree $n$ over $L$ (denoted by $(a, b$; $n, L, \zeta)$ in [2]; see [2, Lemma 1, p. 78]), hence

$$
A_{2} \sim \operatorname{Cor}_{L / F}(a, b) \text {. }
$$

We complete the proof by showing that the corestriction of $(a, b)$ is a symbol algebra: this readily follows from the "projection formula" [2, Theorem 7, p. 88] if $b \in F$, so we can assume $b \notin F$. (Note that $a \notin \dot{F}$, or else the lemma would imply $a \in F^{x n}$, a contradiction.) Since $[L: F]=2$, one can then find $a^{\prime}, b^{\prime} \in F$, both nonzero, such that $a a^{\prime}+b b^{\prime}=0$ or 1 . Then $\left(a a^{\prime}, b b^{\prime}\right) \sim 1$, so that

$$
(a, b) \sim\left(a, b^{\prime}\right)^{-1} \otimes\left(a^{\prime}, b b^{\prime}\right)^{-1} \text {. }
$$

Taking the corestriction of both sides, we get by the "projection formula":

$$
\operatorname{Cor}_{L / F}(a, b) \sim\left(N_{L / F}(a), b^{\prime}\right)^{-1} \otimes\left(a^{\prime}, N_{L / F}\left(b b^{\prime}\right)\right)^{-1} \text {. }
$$

The lemma shows that the first factor on the right-hand side is trivial, hence $\operatorname{Cor}_{L / F}(a, b)$ is similar to a symbol algebra. Q.E.D.

REMARK. This proof can be readily adapted to the case where char $F=n$ (prime), by replacing symbols $(a, b)$ by $n$-symbols $[a, b)$ : one first shows that $K=L(\alpha)$ for some $\alpha$ such that $a:=\alpha^{n}-\alpha \in L$ and $\operatorname{Tr}_{L / F}(a)=u^{n}-u$ for some $u \in F$; the same arguments as above then show that it suffices to prove that $\operatorname{Cor}_{L / F}[a, b)$ is a symbol algebra (for any $b \in L$ ), and this follows from a decomposition:

$$
\operatorname{Cor}_{L / F}[a, b) \sim\left[\operatorname{Tr}_{L / F}(a), b^{\prime}\right) \otimes\left[a^{\prime}, N_{L / F}\left(b^{\prime \prime}\right)\right) \text {. }
$$

\section{REFERENCES}

1. A. A. Albert, A note on normal division algebras of prime degree, Bull. Amer. Math. Soc. 44 (1938), $649-652$.

2. P. K. Draxl, Skew fields, London Math. Soc. Lecture Note Series 81, Cambridge Univ. Press, Cambridge, 1983.

3. L. H. Rowen and D. J. Saltman, Dihedral algebras are cyclic, Proc. Amer. Math. Soc. 84 (1982), $162-164$.

Université de Mons-Hainaut, B-7000 Mons, Belgium

Universite Catholique de Louvain, B-1348 Louvain-la-Neuve, Belgium 